\title{
Extraction of surgical clip-induced "lollipop" choledocholithiasis
}
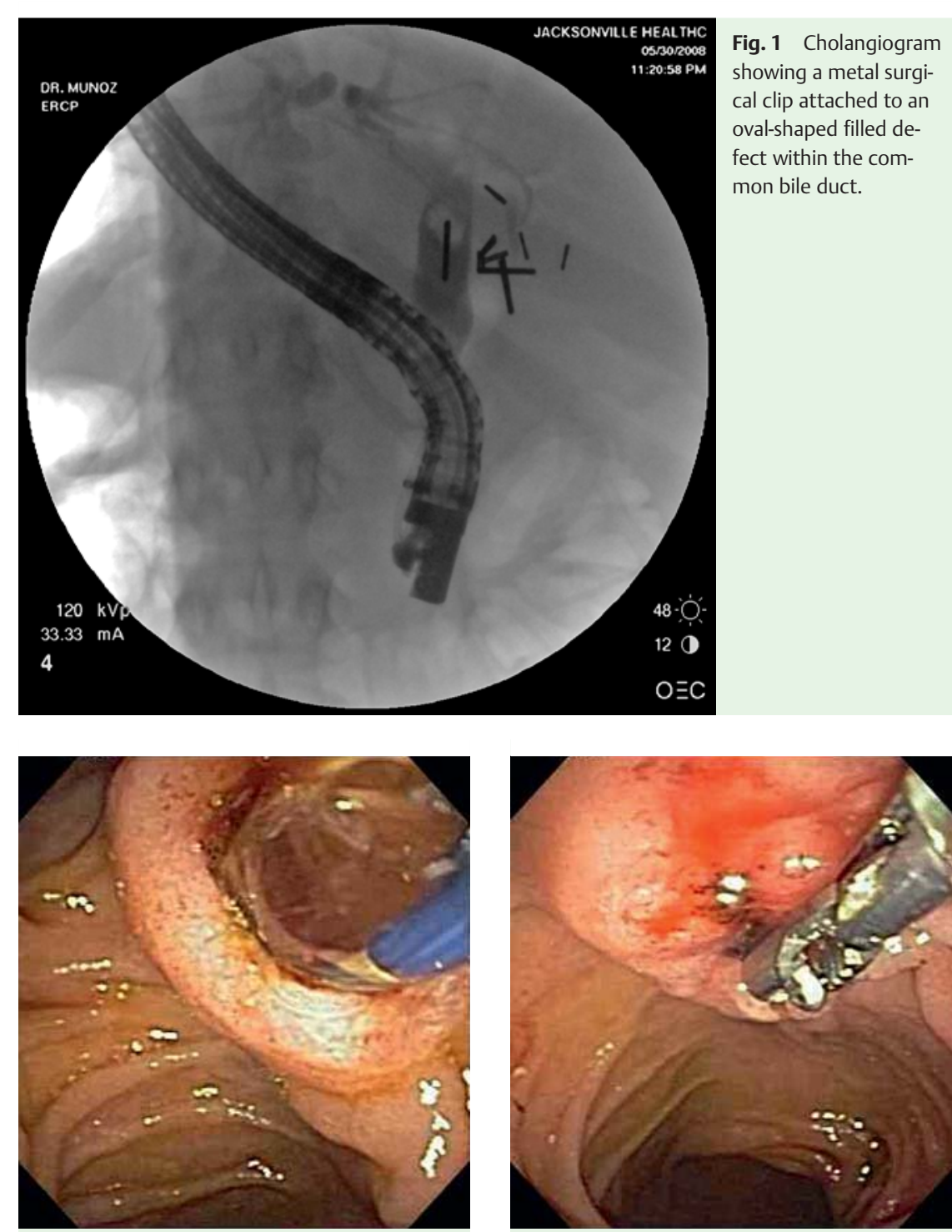

Fig. 2 Endoscopic image of the dilation balloon through the ampulla of Vater.

Foreign bodies arising from migrated surgical clips in the common bile duct (CBD) are rare. These usually occur following a cholecystectomy, with gallstone formation around the migrated surgical clip [1 - 3]. We describe the first successful endoscopic extraction, without sphincterotomy, of choledocholithiasis induced by a surgical clip. With the use of a balloon dilator and rat-tooth forceps, a complex CBD stone/foreign body was removed without complications.

A 57-year-old woman presented with a 1day history of abdominal pain. On exami-
Fig. 3 Rat-tooth forceps grasping the clip in the duodenal lumen during the extraction procedure.

nation there was no fever, chills, or urine/ stool discoloration. Her vital signs were normal except for elevated blood pressure. There were no signs of scleral icterus or chronic liver disease. The abdominal examination was significant only for epigastric tenderness on palpation. Multiple surgical clips in the right upper quadrant, consistent with a history of cholecystectomy 30 years earlier, were seen on plain abdominal radiography. An abdominal computed tomography (CT) scan revealed mild intra/extrahepatic ductal dilatation.

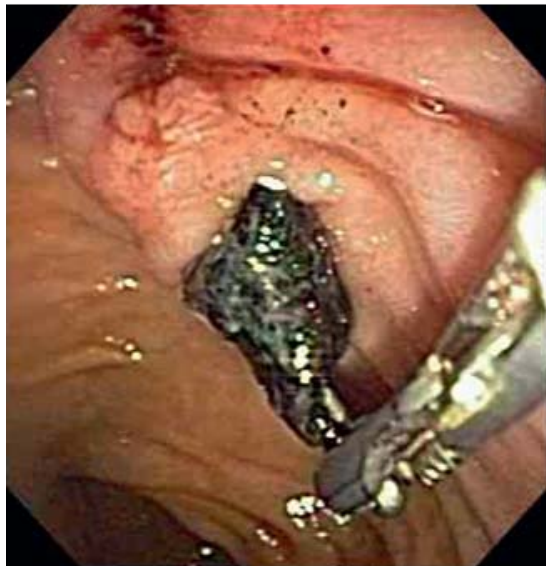

Fig. 4 Rat-tooth forceps grasping the clip and the attached stone in the duodenal lumen during the extraction procedure.

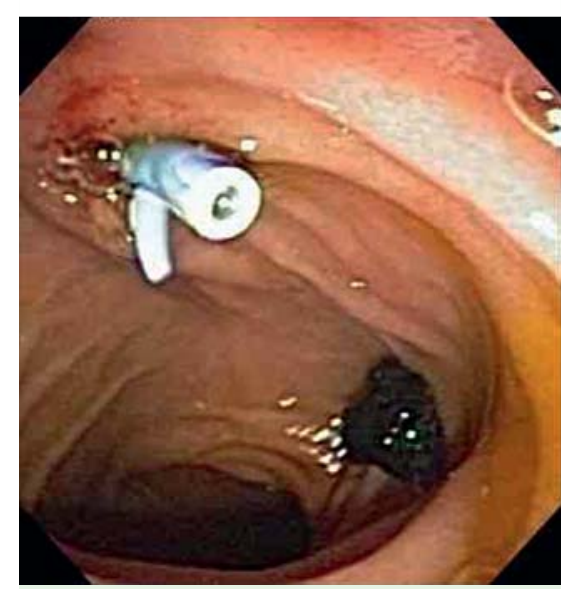

Fig. 5 The extracted stone and attached clip within the duodenal lumen with a new biliary stent in place.

However, the patient's condition improved spontaneously and she was discharged with instructions to return on reoccurrence of pain or fever. After 4 weeks, the patient returned and repeat abdominal CT revealed increased dilatation of the CBD. Emergent endoscopic retrograde cholangiopancreatography (ERCP) demonstrated a single CBD defect with multiple surgical clips surrounding the duct. During balloon dredging, fluoroscopy suggested migration of one of the clips. The patient was transferred to our institution for repeat ERCP to determine the clip's location and, if possible, for extraction endoscopically. 
ERCP demonstrated a dilated CBD (14 mm) and a luminal defect with multiple surgical clips at the level of the bifurcation; initially the clips were not mobile ( Fig. 1 ). The sphincter was dilated using a dilating balloon ( Fig. 2). The CBD was then swept with a retrieval balloon and an ovalshaped defect with an attached surgical clip was observed migrating distally. As the defect approached the papilla, further migration was not possible. The balloon was deflated and removed, and, because of the clip's orientation (distally in the CBD), a rat-tooth forceps was introduced inside the papilla to grasp it under fluoroscopic guidance, and the "object" was extracted carefully into the duodenum ( $\bullet$ Figs. 3-5). The clip and attached stone measured approximately $12 \times 15-18 \mathrm{~mm}$ ( $\bullet$ Figs. 4, 5). A repeat cholangiogram immediately afterward indicated a clear CBD. Following removal, no further complications were reported.
In conclusion, in patients with biliary colic, pancreatitis, and/or cholangitis after cholecystectomy, migration of a surgical clip or clip-induced choledocholithiasis should be considered. Although ERCP with sphincterotomy remains the preferred diagnostic/treatment modality, if circumstances preclude or limit sphincterotomy, the method described here permits extraction, using available accessories.

Endoscopy_UCTN_Code_TTT_1AR_2AG

\section{J. C. Munoz ${ }^{1}$, I.Rascon-Aguilar ${ }^{1}$,}

L. R. Lambiase' , Z. T. Awad ${ }^{2}$, K. J. Vega ${ }^{1}$

1 Division of Gastroenterology, University of Florida College of Medicine/Jacksonville, Jacksonville, Florida, USA

2 Department of Surgery, University of Florida College of Medicine/Jacksonville, Jacksonville, Florida, USA

\section{References}

1 Dolay K, Alis H, Soylu A et al. Migrated endoclip and stone formation after cholecystectomy: a new danger of acute pancreatitis. World J Gastroenterol 2007; 13: 6446-6448

2 Attwell A, Hawes R. Surgical clip migration and choledocholithiasis: a late, abrupt complication of laparoscopic cholecystectomy. Dig Dis Sci 2007; 52: 2254-2256

3 Steffen $M$, Kronsbein $H$, Wesche L. Metal clip as a nidus for formation of common bile duct stone following laparoscopic cholecystectomy. Z Gastroenterol 2007; 45: $317-$ 319

\section{Bibliography}

DOI $10.1055 / \mathrm{s}-0029-1215367$

Endoscopy 2010; 42: E15-E16

(c) Georg Thieme Verlag KG Stuttgart · New York . ISSN 0013-726X

Corresponding author

K. J. Vega, MD

Division of Gastroenterology

University of Florida College of Medicine/

Jacksonville

4555 Emerson Expressway

Jacksonville

FL 32207

USA

Fax: +904-633-0028

kenneth.vega@jax.ufl.edu 